\section{Commentary: With great power comes great responsibility- assessing the validity of propensity score methods}

\author{
Oliver K. Jawitz, MD, MHS, and \\ Vignesh Raman, MD, MHS
}

Randomized controlled trials are the gold standard study design for determining the effectiveness of an intervention including the causal relationship between interventions and outcomes. The advantages of randomization are numerous but primarily involve the elimination of bias by balancing known and unknown prognostic factors among treatment groups. In reality, performing a randomized controlled trial is not always feasible or ethical, and thus we must often rely on observational studies to test clinical hypotheses. This is particularly true when it comes to studying the surgical management of diseases, as these interventions tend to be complex, costly, and difficult to standardize.

When we make causal inferences based on observational data, we are inherently relying on a set of assumptions regarding the magnitude of potential sources of bias that may influence the observed relationship between treatment and outcome. The complexity and sophistication of statistical techniques available have greatly increased and researchers are now able to perform "quasi-randomization" using observational data in an effort to minimize bias. In the cardiothoracic surgery literature, this has frequently taken the form of propensity score matching or weighting. While propensity scores can be a powerful tool in the statisticians' armamentarium to reduce bias, these methods also rely on a set of assumptions that are not infrequently violated. In this issue of the Journal, Baiocchi and colleagues $^{1}$ present an expertly crafted and easily

\footnotetext{
From the Department of Surgery, Duke University Medical Center, Durham, NC. Disclosures: The authors reported no conflicts of interest.

The Journal policy requires editors and reviewers to disclose conflicts of interest and to decline handling or reviewing manuscripts for which they may have a conflict of interest. The editors and reviewers of this article have no conflicts of interest.

Received for publication Sept 22, 2020; revisions received Sept 22, 2020; accepted for publication Sept 23, 2020; available ahead of print Sept 28, 2020.

Address for reprints: Oliver K. Jawitz, MD, MHS, Department of Surgery, Duke University School of Medicine, Box 3443,Durham, NC 27710 (E-mail: oliver.jawitz@ duke.edu).

J Thorac Cardiovasc Surg 2022;163:754-5

$0022-5223 / \$ 36.00$

Copyright (c) 2020 by The American Association for Thoracic Surgery

https://doi.org/10.1016/j.jtcvs.2020.09.092
}
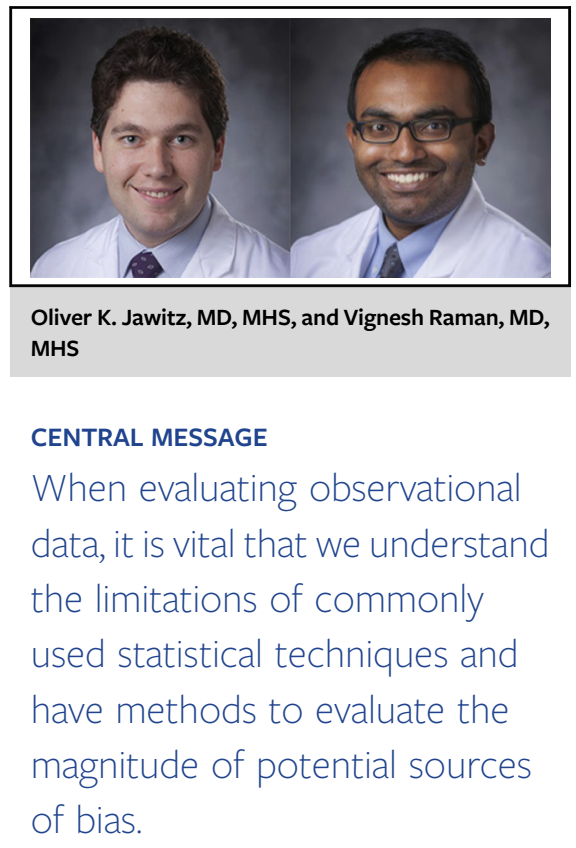

approachable primer regarding the use of modern formal sensitivity analyses to quantify the resiliency of these studies' findings to bias.

As described in their manuscript, the validity of studies based on propensity scores relies on their assumption of strongly ignorable treatment assignment-essentially the observed covariates are sufficient to determine which treatment a particular subject will receive. The degree to which a particular study's findings are resilient to violations of this assumption is what we can assess using techniques such as the gamma sensitivity analysis. When interpreting these sensitivity analyses, it is essential to understand what they do and do not convey. Importantly, these techniques allow you to quantify, in a graded fashion, how sensitive the study results are to potential assumption violations, but they do not actually evaluate whether these violations occurred.

While the aforementioned techniques allow you to assess a study's internal validity, they do not provide any meaningful description of its external validity - the degree to which the findings would apply to a broad, real-world population. ${ }^{2}$ In the cardiothoracic surgery literature, we frequently see propensity score matching techniques employed where a small subset of the overall study population is analyzed to determine the relationship between treatment and outcome. While the causal relationship observed likely holds true for the small group of subjects examined, it is often questionable whether similar conclusions can be drawn for larger 
populations from diverse settings. When we choose to employ statistical techniques involving propensity scores, as well as evaluate studies that use these methods, we must evaluate both internal and external validity to ensure our conclusions drawn from observational data are robust and reliable.

\section{References}

1. Baiocchi M, Woo YJ, Chiu P, Goldstone AB. The role and significance of sensitivity analyses in enhancing the statistical validity of clinical studies. $J$ Thorac Cardiovasc Surg. 2022; 163:749-53.

2. Freemantle N, Marston L, Walters K, Wood J, Reynolds MR, Petersen I. Making inferences on treatment effects from real world data: propensity scores, confounding by indication, and other perils for the unwary in observational research. $B M J$ 2013:347:f6409.
See Article page 749.

\section{Commentary: Sensitivity analyses: Mitigating the problem of garbage in, equals garbage out?}

\section{Anusha Jegatheeswaran, MD, PhD, FRCSC}

In this issue of the Journal, Baiocchi and colleagues ${ }^{1}$ present the importance of sensitivity analyses in improving the validity and diminishing the bias inherent in observational studies. Of course, the gold standard in outcome evaluation, due to its ability to eliminate bias in studies, is the implementation of randomized controlled trials to answer questions where the determination of causality is the primary goal. However, in cardiac surgery, there are many questions for which a randomized controlled trial is simply not possible, practical, or in some cases ethical. This may occur due to a lack of equipoise, the inability of different sites to carry out techniques with which they are unfamiliar, or secondary to exceedingly small patient numbers, which is often the case in the domain of pediatrics. As such, we are often left to rely on observational data and quasiexperimental designs to provide us with the best possible results to inform important decisions regarding patient care.

Those unfamiliar with performing statistical analyses themselves may not truly appreciate the number of "decisions" that must be made to get from a raw data set to a final

\footnotetext{
From the Division of Cardiovascular Surgery, Department of Surgery, Hospital for Sick Children, University of Toronto, Toronto, Ontario, Canada.

Disclosures: The author reported no conflicts of interest.

The Journal policy requires editors and reviewers to disclose conflicts of interest and to decline handling or reviewing manuscripts for which they may have a conflict of interest. The editors and reviewers of this article have no conflicts of interest.

Received for publication Sept 20, 2020; revisions received Sept 20, 2020; accepted for publication Sept 23, 2020; available ahead of print Sept 28, 2020.

Address for reprints: Anusha Jegatheeswaran MD, PhD, FRCSC, Division of Cardiovascular Surgery, Hospital for Sick Children, 555 University Ave, Toronto, Ontario M5G 1X8, Canada (E-mail: anusha.jegatheeswaran@utoronto.ca).

J Thorac Cardiovasc Surg 2022;163:755-6 $0022-5223 / \$ 36.00$

Copyright (C) 2020 Published by Elsevier Inc. on behalf of The American Association for Thoracic Surgery

https://doi.org/10.1016/j.jtcvs.2020.09.086
}

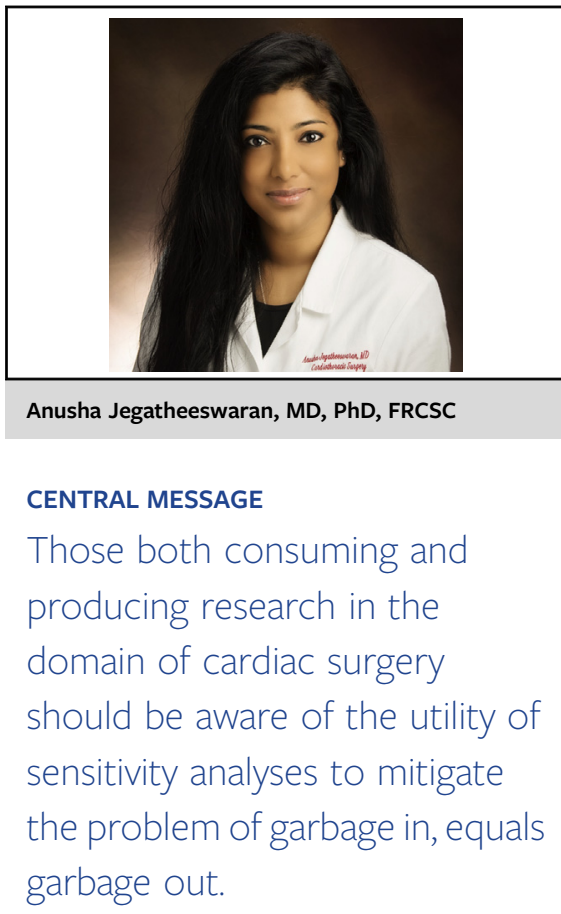

output with conclusions. These decisions can have a wide spectrum of influence on the results of any study and may range from something as simple as how to split patients into comparison groups, to more complex decisions involving the specific nature of how study variables are created, and determination of the variables used when matching patients. While it may seem like these are tiny decisions of little consequence, ultimately it is this multitude of decisions which, when compounded, can cause the results to lie farther and farther from the truth. This is in addition to issues that may arise from small datasets or poor data quality.

Similarly, those without a statistical background may not be aware that all statistical models are based on assumptions. As such, the validity of the conclusions that can be drawn from these models is highly dependent on the degree to which the assumptions are met. The primary drawback of 